\title{
Model of Bayesian tangent eye shape for eye capture
}

\begin{abstract}
Iris recognition system captures an image of an individual's eye. In addition, the process of segmentation, normalization and feature extraction is followed by the iris of an eye image in the system. Using the algorithms proposed by J. Daugman, Iris recognition system has significantly improved over the last decade, and it has been used in so many practical applications. However, some difficulties related to Iris position and movement are still to be improved. To overcome these difficulties one can enhance the image acquisition process. Obtaining a method in extracting quality of eye images automatically from the video stream is the main area of interest in this study. Besides, a Bayesian inference solution called Bayesian Tangent Eye Shape Model (BTESM) was suggested depending on estimation of tangent shape. During image acquisition, constraints on the position and motion of the subjects can be decreased owing to this approach. Owing to maximum a posteriori estimation, we can identify similarity transform coefficients as well as the eye shape parameters in BTESM. To apply the maximum a posteriori procedure, tangent Eye shape vector was considered the state of the model which is hidden and expectation maximization depending on searching algorithm was adopted. Hence, after being tested and matched to future studies, the acquisitioned eye image has been proved to be adequate for Iris recognition system.
\end{abstract}

Keyword: Eye detection; Iris recognition based on video; Iris recognition on the move; Bayesian tangent eye shape model; Estimation of eye position 